\title{
KEPLER CONCORDIA: DESIGNING AN IMMERSIVE MODULAR MUSICAL AND SCIENTIFIC INSTRUMENT USING NOVEL BLOCKCHAIN AND SONIFICATION TECHNOLOGIES IN XR
}

\author{
Kelly Snook, Tarik Barri, Joachim Goßmann, Jason Potts, Margaret Schedel, Hartmut Warm \\ University of Brighton \\ Fab Lab, EM13 Heavy Engineering, Lewes Road, Brighton, BN2 4GJ \\ K. Snook@brighton.ac.uk
}

\begin{abstract}
This paper describes the first steps in the creation of a new scientific and musical instrument to be released in 2019 for the 400th anniversary of Johannes Kepler's Harmonies of the World, which laid out his three laws of planetary motion and launched the field of modern astronomy. Concordia is a musical instrument that is modularly extensible, with its first software and hardware modules and underlying framework under construction now. The instrument is being designed in an immersive extended-reality (XR) environment with scientifically accurate visualizations and datatransparent sonifications of planetary movements rooted in the musical and mathematical concepts of Johannes Kepler [1], extrapolated into visualizations by Hartmut Warm [2], and sonified. Principles of game design, data sonification/visualization optimization, and digital and analog music synthesis are used in the 3D presentation of information, the user interfaces (UX), and the controls of the instrument, with an optional DIY hardware "cockpit" interface. The instrument hardware and software are both designed to be modular and open source; Concordia can be played virtually without the DIY cockpit on a mobile platform, or users can build or customize their own interfaces, such as traditional keyboards, button grids, or gestural controllers with haptic feedback to interact with the system. It is designed to enable and reward practice and virtuosity through learning levels borrowed from game design, gradually building listening skills for decoding sonified information. The frameworks for uploading, verifying, and accessing the data; programming and verifying hardware and software module builds; tracking of instrument usage; and managing the instrument's economic ecosystem are being built using a combination of distributed computational technologies and peer-to-peer networks, including blockchain and the Interplanetary Filesystem (IPFS). Participants in Concordia fall into three general categories, listed here in decreasing degrees of agency: 1) Contributors; 2) Players; and 3) Observers. This paper lays out the broad structure of Concordia, describes progress on the first software module, and explores the creative, social, economic, and educational potential of Concordia as a new type of creative ecosystem.
\end{abstract} available at http://creativecommons.org/licenses/by-nc/4.0

\section{INTRODUCTION AND HISTORICAL CONTEXT}

2019 marks the four-hundredth anniversary of Kepler's publication Harmonices Mundi (Harmonies of the World), a treatise on geometry, astronomy and music [1]. The book, commissioned by the City of Linz and published in 1619, details his first three laws of planetary motion, as well as his process of discovering them. The three laws, now well known, were not initially accepted, even by contemporary astronomer, Galileo, because they conflicted with accepted theological world-views based on the geocentric model of Ptolemy:

1. All planets move about the Sun in elliptical orbits, having the Sun as one of the foci

2. A radius vector joining any planet to the Sun sweeps out equal areas in equal lengths of time

3. The squares of the sidereal periods (of revolution) of the planets are directly proportional to the cubes of their mean distances from the Sun

Four specific aspects of Kepler's approach were new or unique and bear mentioning. First, his writings make clear that he was primarily motivated by exposing and making accessible the harmonic order that he considered to be God's organizing principle [3]:

I feel carried away and possessed by an unutterable rapture over the divine spectacle of the heavenly harmony.

This, in itself, was not unique in the early 17 th century. But his dogged insistence on understanding and publicly exploring deep spiritual principles, rather than supporting any of the rival Christian dogmas of his day, led to all of them rejecting him and he was left to seek spiritual satisfaction in astronomy [4]:

...You see how near I come to truth. As often as this happens, how can you doubt that I amply shed tears! For God knows, this a priori method [the scientific method developed by him and Galileo] serves to improve the study of the movements of the celestial bodies; we can put all our hopes into it if others cooperate who have observations at their disposal... I am eager to publish soon, not in my interest, dear teacher... I strive to publish in God's honor who wishes to be recognized from the book of nature. But the more others continue in these endeavors, the more I shall rejoice; I am not envious of anybody. This I pledged to God, this is my decision. I had the intention of becoming a theologian. For a long 
time I was restless: but now see how God is, by my endeavors, also glorified in astronomy.

Rejection from his native Lutheran Church caused him deep personal grief and sorrow. He found solace from this sorrow in his new astronomy and mathematics:

If God fulfills these wishes, my mathematics will always be ready to offer delight... and give relief from sorrow either in the astronomical practices or in the contemplation of the heavenly works of the harmonies of the universe.

Second, in true Renaissance style, in addition to deep mathematical, astronomical, and theological knowledge, Kepler also possessed profound understanding of the most recent music theory of his day. At a critical juncture in his development, he was inspired by the work of Galileo's father, Vincenzo Galileo [5], who examined music theory from a broad historical and newly experimental perspective, with special attention paid to early Greek theory and tuning systems. While the Pythagorean idea of the "Music of the Spheres" was widespread and even long-embedded in Christian dogma, Kepler approached music as a tool for physical investigation of reality with unprecedented rigor and enthusiasm.

Third, Kepler, during his tenure as Imperial Mathemetician to the Holy Roman Emperor Rudolph, had sole access to the most comprehensive and accurate data ever collected in the meticulously compiled astronomical observations of his predecessor, Tycho Brahe. His refusal to accept previously-ignored or unknown discrepancies between observed data and prevailing models, whether Ptolemaic or Copernican, eventually led to his breakthroughs in discovering the correct mathematics reflected in his three laws. No one before him had so rigorously pursued precise agreement between theory and observation, one of his principal contributions to the scientific method.

Finally, the fourth and perhaps most breathtaking and admirable aspect of Kepler's approach was his ardent prayer, granted several times over, to be relieved of any attachment to ideas that stood in the way of the truth he so tirelessly pursued. Most notably, when the unexpected mathematical truths of his three laws finally dawned on him, he was forced to let go of his cherished, physically and philosophically beautiful, early model of the solar system, consisting of nested platonic solids, which had launched his career and sustained his passionate inquiry for decades. His own discoveries undermined not only his contemporaries's worldviews, but his own favourite ideas. Coupled with this was his ability and willingness to extrapolate truths to the broader human condition:

While I struggle to bring forth this process into the light of human intellect by means of the elementary form customary with geometers... may He prevent the darkenss of our mind from bringing forth in this work anything unworthy of His Majesty... Holy Father, keep us safe in the concord of our love for one another, that we may be one... just as through the sweetest bonds of harmonies Thou hast made all Thy works one... as Thou didst erect the heavens themselves out of harmonies.

In light of this brief examination of Kepler's approach, it is evident that modern science, by distilling his contributions to their most basic mathematics, does a disservice to his multidimensional, uniquely multidisciplinary pursuit of truth, which called on every possible human faculty and begs further research. Kepler, himself, issued a call to future musicians to investigate [1]:
...There is need for louder sound while I climb along the harmonic scale of the celestial movements to higher things where the true archetype of the fabric of the world is kept hidden. Follow after, ye modern musicians, and judge the thing according to your arts, which were unknown to antiquity.

Although it is unorthodox in modern times to supply such a detailed religious and historical account in a scientific paper, it is necessary to demonstrate the various ways that Concordia is a direct response to this call by Kepler. Far beyond a simple academic exercise in data sonification, it is a scientific and musical instrument designed explicitly to celbrate and make accessible Kepler's ideas as an immersive, visceral, educational experience. The creation of a modular, customizable musical instrument, as opposed to a musical performance or recorded piece of music or art, gives agency to each player to explore deeply their own truths. Building such an experience requires the application of modern astrophysical data, computational architectures and music technology to modern datasets, to create a comprehensive, scientifically accurate solar system model with abstract representations of geometric harmonies superimposed. Both the hardware and software for Concordia will be open source, developed in modules, and uploaded to the Concordia blockchain and other distributed computational infrastructures. The launch in 2019 will feature live streamed virtual-reality compositions by musicians that can be observed from anywhere in the world.

\section{A NEW TYPE OF MUSICAL INSTRUMENT}

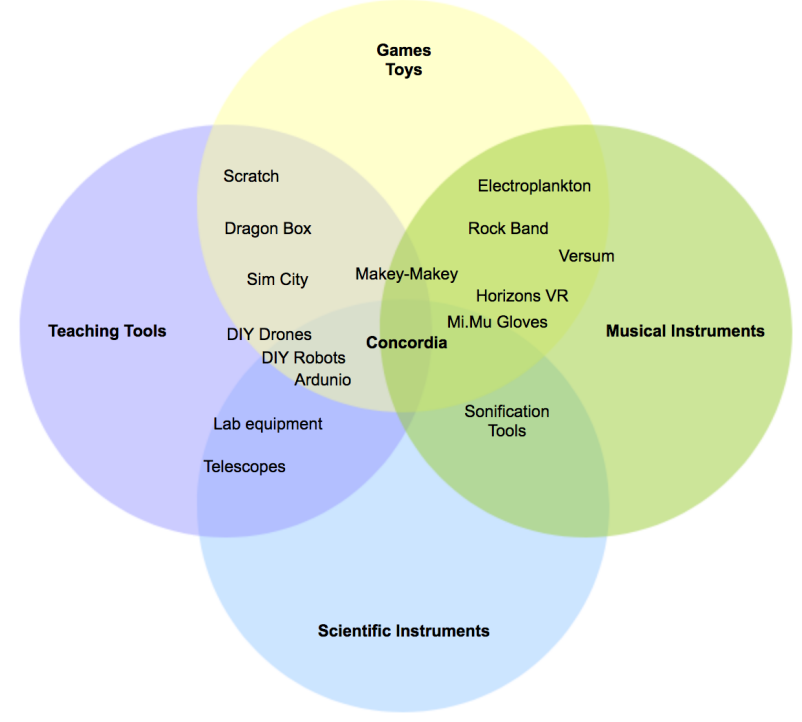

Figure 1: The overlapping arenas of Concordia and some examples of other established instruments, tools, and games

Lying at the intersection of music, science, technology, gaming, and pedagogy (Figure 1), Concordia draws elements from all in equal measure. It resembles a game-like interactive, immersive installation in which single or multiple players navigate through one or more datasets. The instrument's visualization/sonification software and hardware are both modular, similar to the pedals and 
effects of a live guitarist, the modules of a modular synthesizer, or custom objects/patches in MaxMSP or Pure Data. Concordia provides the computational and philosophical framework upon which modules can be built, played, and observed, and has three main elements:

1. Interactive data sources - players choose which physical phenomena they would like to explore and are presented with a wide array of parameters to vary, including time, selection of planets, moons, etc. (specific controls are designed by module creators). Concordia data and the algorithms comprising each module are stored in distributed, syncronized peer-to-peer networked and verified databases;

2. Immersive audiovisuals, haptics - Players explore via VR headset, AR glasses, or 360 projection of a dynamic solar system to scale and other geometric objects highlighting harmonic relationships. Spatialized sound is either rendered binaurally to headphones or realized by a multichannel loudspeaker array;

3. Hardware controls - Controls can be minimal, such as through a tablet or computer, or can expand to match the imagination of players through an optional array of traditional or non-traditional controls for interaction with the data and navigation through space and time.

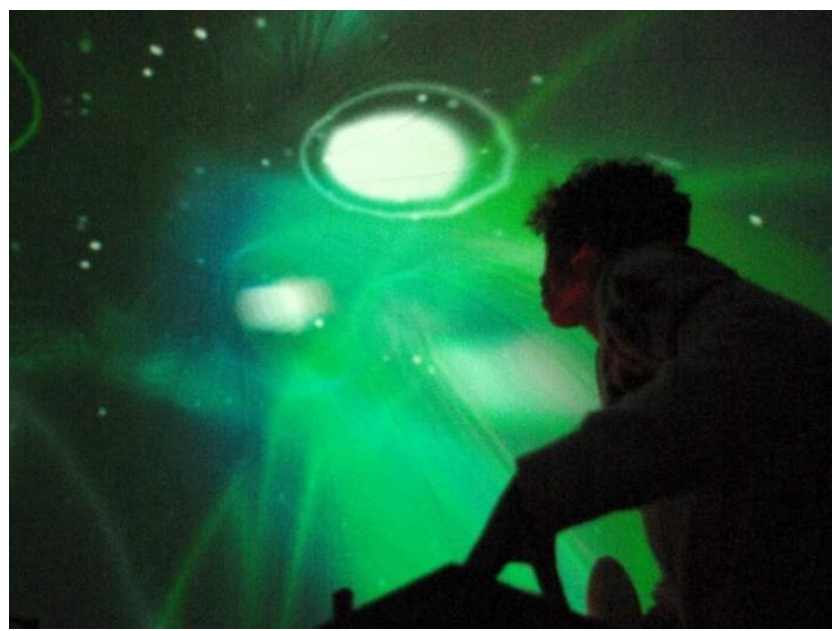

Figure 2: Tarik Barri playing a composition in Versum

Figure 2 shows Tarik Barri interacting with his Versum [6] audiovisual composition environment inside a projection dome. The initial Concordia module prototype uses Versum to display visualizations and sonifications of planetary data. Whereas Versum allows a composer unlimited creative freedom in sound and visual design, all sounds and visuals in Concordia modules are generated by planetary data. The release version of Concordia will behave like a game. It will use rewards and levels of play to train players gradually in the new listening and control skills required to understand, explore, and eventually achieve virtuosity with the instrument. Its modular, open-source, DIY hardware designs and distributed architecture of software invite participation globally and reduce barriers present in traditional music creation and collaboration.

Participants in Concordia fall into three general categories, listed here in decreasing degrees of agency:
1. Contributors - those who design, program, create and upload new sonification or visualization modules, new DIY hardware control interface designs for others to download and build, or new compositions within existing modules. Contributors could also be people who facilitate new use cases for Concordia, such as presets or ways of playing Concordia that can be used in teaching curricula.

2. Players - those who use the instrument controls actively to choose which Concordia modules to play, to choose which data within given modules to play, and then to navigate, manipulate, and explore the data and their mappings into the 4D immersive world of sound, visuals, and haptics. Players have the option of inviting others to observe their live explorations, and/or recording their compositions back into Concordia for others to play and remix.

3. Observers - those who "ride along" with someone else who is playing live or with a recording inside Concordia. This category of participant can act as an audience or beneficiary of experiences others are creating.

These three categories of Concordia participants together form a community that functions as a creative economy and a collaborative ecosystem based on built-in, blockchain-based, communitydefined smart contracts for tracking the value of contributions and use. This allows Concordia to serve as a socioeconomic experiment as it grows and evolves.

\subsection{Astronomical Sonifications and Visualizations}

There have been numerous sonifications of planetary movement and other astronomical data $[7,8,9,10,11]$, and sonifications directly referecinng Kepler's work, notably Laurie Spiegel's Kepler's Harmony of the Worlds, which was included on Voyager's Golden Record [12], and Rodger's and Ruff's Realization for the ear of Johannes Kepler's astronomical data from Harmonices Mundi" [13]. These sonifications owe a conceptual debt to Pythagoras's Music of the Spheres, a philosophical conceit that sought to describe the movement of the sun, moon, and planets using harmonic proportions. The Music of the Spheres was not thought to be literally audible, but rather to be a harmonic, mathematical or religious concept; in later Christian reinterpretation of the Music of the Spheres, instrumental music was thought to exist solely to make audible the other two types of inaudible music: the music of the spheres and the music of the human spirit.

As discussed earlier, Kepler was highly influenced by the idea of the Music of the Spheres, and he sought a rational explanation of the movements of the planets, of which only six were known in his time. After exhausting, unsatisfied, sonifications of planetary orbital distances and other static parameters, he progressed to more abstract and complex relationships between angular velocities of the planets during particular times in their orbits, such as aphelion and perihelion. Over the centuries since, the solar system has inspired sonifications too numerous to adequately survey here. Many recent solar system sonifications translate direct planetary measurements such as position, size, density, composition, etc. into controls for the sound. Quinton et al. [14] discuss important issues that arise in the context of their research into effective solar system sonification methods, if they are to be used for anything other than mere entertainment.

Through sonification, abstract concepts like planetary movements, can be made more tangible and 
comprehensible to the general public. Since many sonifications have been created as artistic pieces a certain degree of scepticism from scientists has made them wary of using sonification as a scientific tool. Barrass refers to the conflict between the more traditional scientific view with relation to data analysis and the new view, which embraces the advantages of the human auditory system and its cultural significance. There is a fine line between sonification as a means of scientific exploration and merely being perceived as a popular mass media marketing tool.

Considering the large amounts of data involved in astrophysics the use of sonification in this field can be explored in more detail. The difficulty arises when trying to find the right balance between artistic input and sonifications that are usable in scientific data analysis [14].

The solar system is a structure of significant spatial extent and complexity offering a seemingly infinite variety of possible perspectives. Kepler was interested in the relationships between the heavenly entities, as opposed to any individual planet considered alone, so it is these relationships that are the focus of the first Concordia modules. In this arena, Hartmut Warm has created a body of work $[2,15]$ that extrapolates and extends Kepler's ideas by representing them visually and examining the geometric archetypes intrinsic to dynamic planeary relationships.

Warm has created a computer application based on open hybrid observational and theoretical ephemerides models (e.g. [16]) that visualizes a wide variety of geometric, spirograph-like figures discussed in the text. Using his app, one can calculate and draw astronomical objects and events, such as relative positions, velocities, conjunctions, and oppositions. The first Concordia module prototype then uses the strings of numbers output from Warm's code to generate 3D visual and sonic objects in Versum for module creators to experiment with mappings to waveforms, envelope shapes, filters, colors, visual shapes, and time-based effects.

The approach of Concordia, mirroring Kepler's, is to minimize the imposition of pre-formed ideas or musical constructs as much as possible from the sonification algorithms, with the goal of allowing people to find mappings that naturally expose the symmetry, beauty, harmony and dissonance - the "musicality" - of the physics. For example, a Concordia contributor, as they program a new module, might favor, when choosing sonic mappings for the lines in Figure 3, mapping directly to frequency space rather than mapping to "pitch" via pre-selected notes in a pre-selected scale. In short, minimizing subjective aesthetic impositions from the mappings will enable maximum freedom to create subjective, aesthetically rich results in the exploratory playing of the instrument. Sophisticated, model-based sonification algorithms will be favored over direct audification, allowing for ever richer layering of information into the sounds being created. The simple, direct mappings of planetary information may be used as anchors for the subtler details that characterize the data, embracing rather than neglecting those particularly interesting imperfections or deviations from averages. For example, sound designers know that to make a truly interesting sound, things like Low Frequency Oscillation (LFO), filter cut-off frequency changes, Attack/Decay/Sustain/Release Envelope control, and thousands of other variables in synthesis are critical considerations. Eventually, any given combination of Concordia modules could employ
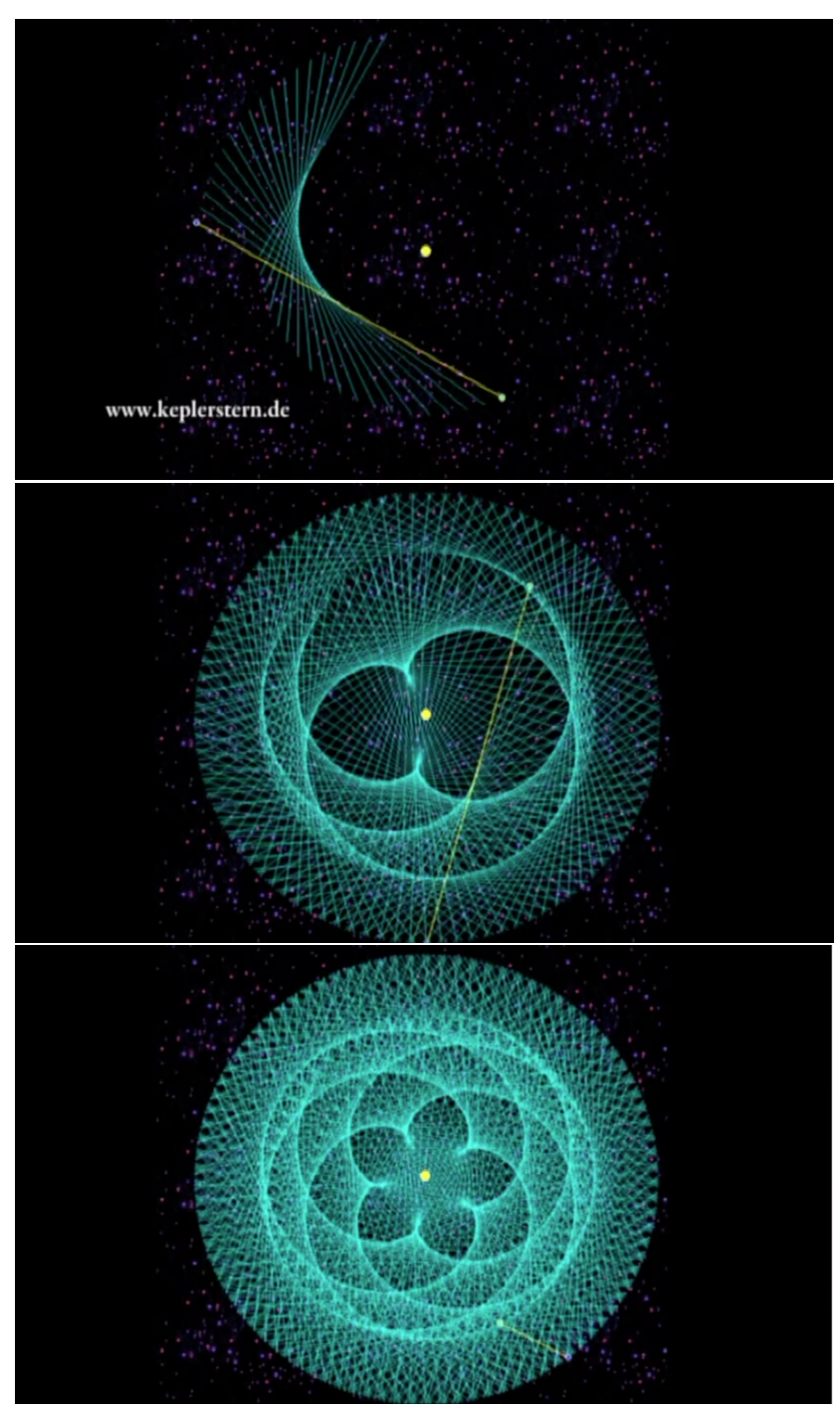

Figure 3: Screenshots of Hartmut Warm's Keplerstern program displaying linklines between Venus and Earth every 4 days over a period of 100 years.

thousands, if not hundreds of thousands, of algorithmic, modelbased sonification and visualization mappings in a form that is designed to grow and evolve through open-source contributions to the code base. Composers will choose which of those relationships are pleasing to their ears and how to trigger or manipulate those in time. As Scaletti notes, Sonification $\neq$ Music [17]; the complexity and number of possible mappings will ensure that Concordia is an expressive musical instrument.

One primary consideration will be modularity and the flexibility of mapping algorithms, finding the optimimum balance between the limitations of scientific accuracy and integrity versus the freedom of sonic exploration and expression. Another consideration in module design will center around deciding which parameters to map to the visual domain and which to map to the sonic or haptic domains. Concordia's hardware control interfaces and modules will be similarly flexible, with the ability to manipulate the data through a wide range of controllers, from simple switches 
on a tablet to complex gestural control such as with motion capture or wearables like the Mi.Mu gloves [18].

\section{2. $\mathrm{AR} / \mathrm{VR} / \mathrm{XR}$ - Visual and Sonic}

Concordia will be built in extended reality (XR), a combination of Virtual Reality (VR) and Augmented Reality (AR), sometimes called "immersive computing." There is not yet widespread agreement on the definitions of boundaries between AR and VR, so it is perhaps more useful to consider VR and AR as two points on a spectrum of the degree to which computer-generated imagery and audio is superimposed upon traditionally-perceived reality [19]. In the case of Concordia, most of the reality represented in the instrument must be computer-generated because its spatial and temporal scales are far outside normal human perception, as Kepler describes in Harmonies of the World:

But whose good will it be to have harmonies between the journeys, or who will perceive these harmonies? For there are two things which disclose to us harmonies in natural things: either light or sound: light apprehended through the eyes or hidden senses proportioned to the eyes, and sound through the ears. The mind seizes upon these forms and, whether by instinct or by astronomical or harmonic radioncination, discerns the concordant from the discordant. Now there are no sounds in the heavens, nor is the movement so turbulent that any noise is made by the rubbing against the ether. Light remains. If light has to teach these things about the planetary journeys, it will teach either the eyes or a sensorium analogous to the eyes and situated in a definite place; and it seems that sense-perception must be present there in order that light of itself may immediately teach. Therefore there will be sense-perception in the total world, namely in order that the movements of all the planets may be presented to sense-perceptions at the same time.

Thus, Concordia itself will present the celestial harmonies to the eyes, the ears, and the sense of physical immersion of the player so their "mind can sieze upon the patterns," using whatever technology is available to the player. Many modules will likely be designed for VR goggles, headphones, and a cockpit of hardware controls, but all will be required to be at least minimally playable on a simple tablet. In any format, Concordia will use both visualizations and sonifications of planetary data, but its primary function is to emphasize the auditory experience and compel musical explorations.

By sonifying information in addition to visualizing it, new pathways are created for mapping data structures that can exploit the human brain's natural expertise for decoding sound, perceiving patterns and correlations, and gaining understanding of the world from it. For people who are both sighted and hearing, using sonification and visualization together provides important corroboration of information between senses that are instinctively used in the world at large [20]. A great deal of time, trial, and error will be required to find elegant mapping algorithms to expose the natural harmonies and dissonances intrinsic in the system, and users will find their own way of playing the instrument, choosing the mappings that please them. Recent advances in technology enable us to combine new sonification concepts with powerful visual cues in an immersive and interactive way that educates through direct, visceral, multi-sensory experience rather than traditional pedagogical methods.

In order to be useful as a musical instrument, Concordia needs to combine interactive affordances with a transparent mapping of scientific data into perceptual domains. In general, pitch (or fundamental frequency) as a musical mapping for information is avoided, in favor of more subtle and emergent sonic parameters such as timbre, envelope shaping, particularly in attack spectral content. Multichannel (3D) spatial sonic information will be correlated exactly with visual spatial information where possible, although additional sonic information may be available without being visible. In the cases where this is done, it is well described to the player.

\subsection{Distributed Peer-to-peer Computing and Community}

The rapidly advancing fields of distributed database computing, collaborative work platforms, media streaming, and blockchain technologies provide exciting and fertile ground for experimenting with large-scale data-driven creative ecosystems. Concordia fully embraces this experimental spirit [21]. While blockchains are most well known in the application of cryptocurrencies, a blockchain is simply a distributed ledger of records linked and secured using cryptography. Any kind of information or contract can be held on a blockchain, and it exists on the network as a continuously reconciled, shared database. Removing the need for trusted third parties such as banks or lawyers, or indeed any large corporations, blockchain technologies are rapidly transforming the socioeconomic landscape in ways that are only beginning to be understood and applied [22]. These technologies present enormous, as yet untapped, potential for large-scale creative collaboration, which can incorporate, as part of the basic infrastructure of a computational musical instrument, the economies and attributions formerly handled by a complex collection of third parties, such as scientific review boards, digital rights management organizations, record labels, and managers.

Blockchain, combined with other distributed database technologies, such as the Interplanetary File System (IPFS), are useful in four different aspects of Concordia implementation:

1. Data storage and verification - Automatically mantaining reliable, accurate, standardized datasets and software modules for the Concordia sonification/visualization modules

2. Attribution and version control of software and hardware modules - Automatically tracking and rewarding or charging contributors, builders, and remixers of hardware and software modules

3. Attribution and source-data-matching for compositions - Tracking composers, performers, and players and ensuring that the compositions are accessing correct versions of data and modules. Rewarding or charging players and observers.

4. Management of live collaborative composition or performance data - Building on existing partially-centralized, partially decentralized technologies such as those used for massively multiplayer online gaming for an optimized playing experience

Blockchain technology can be used to provide permanent (tamperproof, censorship resistant) records of the evolution of a 
distributed instrument as it develops and changes, enabling searchable and verifiable metadata and outputs of the instruments configurations and discoveries that can be accessed and replayed, or reconstructed, by anyone from anywhere. That offers an open science approach to open music (or smart music, a la smart contracts): a world instrument as a global virtual machine.

Clearly, many aspects of the functioning of Concordia will benefit from the affordances of blockchain technologies, while many others would be unnecessarily bogged down by the inefficiencies introduced when requiring cryptography and verification. Where possible, other, more efficient, distributed architectures will be employed. However, blockchain is useful as a mechanism for handling Concordia's embedded economies and social contracts. A system of valuation will be assigned to the various ways of contributing to or benefitting from the Concordia instrument. However this value is defined - whether strictly financial or some other defined value - if that value can be traded, economies emerge. Players will "spend" a very small, fixed amount, to play the instrument or to download the code for modules. Once they create something like a composition or a new code or hardware controller design, they become Contributors. Contributors will earn fixed amounts of value by contributing in any of the ways defined and agreed by the community, such as

1. programming and uploading new sonification or visualization software modules;

2. composing/performing and uploading new pieces that are uploaded for others to experience; and

3. designing and uploading DIY hardware control modules for others to build.

For example, someone who composes a piece might earn enough value to request a new feature from a programmer. Conversely, to play the instrument without uploading anything does not earn value and requires the expenditure of value. Metrics for excellence and algorithms for rewarding excellence will be established such that the most excellent modules, performances, or compositions will earn added value.

As Concordia evolves and grows, and as additional scientific discoveries are made and new data becomes available, the original datasets upon which the instrument is based may change, requiring version tracking that links each performance, composition, and module with the correct versions of each other and the data itself. By careful version control and matching, no composition or performance becomes obsolete and a full history of the evolution of the instrument, and any compositions made with it over time, is accessible, allowing the instrument to be sustainable and all compositions to be easily re-performed [23]. It should be noted that this is a departure from many electronic music frameworks such as digital audio workstations (DAWs) or plug-ins, in which compositions or sessions must be bounced to stems to preserve the details of the performances so that when operating systems or compatibility changes, the sessions can still be heard.

Active exploration of broader socioeconomic and educational implications of this new type of instrument, particular in its distributed ledger aspects, is underway and might best be followed online (e.g. [24]), as progress in these fields outpaces traditional academic research publishing. From an economic perspective, Concordia appears to be the world's first distributed-ledger-based musical instrument, and because of that, it will also be the world's first musical instrument with its own economy. This makes Con- cordia of interest not only to musicians, musicologists and technologists, but also to cultural economists and innovation economists.

Concordia reconceptualises an instrument from a private good or unit of capital to a distributed infrastructure (a protocol), and therefore enables a different economic model, namely instrument as a common pool resource. This works over the protocol and design of the instrument and its settings and other meta-data, and also to the data inputs and outputs. This then changes both the economic incentives at work, the business models that are effective, and the policy settings available. This new economic model also implies new policy models, based on institutional design of protocols and governance. Concordia also illustrates how the creative arts and creative economy can be a vanguard for other parts of the economy or society by early adoption of new technologies and experimental development of the institutions to support them.

\subsection{Concordia in Education}

A full discussion of the educational potential of Concordia, both as a direct element in curriculum design for primary, secondary, and post-secondary or higher education and as a networked installation in museums or institutions is beyond the scope of this paper but bears mentioning as an area being actively explored by the Concordia team.

\section{MODULE 1 - PROTOTYPE}

As a proof of concept, while the underlying computational and social/philosophical frameworks outlined above are being developed, work on a prototype of the first Concordia module has begun. Where possible, existing tools (e.g. the data visualization and sonification envirnoments created by Warm and Barri) are being used for early mapping experiments. As a starting point, two datasets of planetary ephemeris have been generated using standard examples provided in Warm's app, Signatures of the Celestial Spheres [2]:

1. Lines linking Jupiter and Uranus, shown in Figure 4; and

2. Lines linking Venus and Earth drawn every 4 days for 100 years (as shown in Figure 5).

In this simplest of modules, the lines linking the planets form pentagonal and hexagonal figures, respectively, when drawn at the resolution generated in the examples. A MaxMSP patch was created to read in the data, add one dimension of input data control that could be mapped to an OSC controller, then output OSC commands to Barri's Versum audio-visual composition environment to draw the lines and spheres in $3 \mathrm{D}$, apply mappings to audio and visual synthesis, and enable navigation through the data. The objects created in Versum comprise lines and spheres with a large number of time-variable visual and sonic parameters that can be mapped using data-generated OSC commands with an emphasis on visual simplicity and on sonification as the main carrier of geometric and relational information. As the lines between planets are drawn, their mathematical properties are sonified in a way that allows sonic patterns to be revealed gradually, just as the visual patterns are.

The one dimension of data input control chosen was the sampling frequency of the lines drawn over a given time, achieved in the max patch by creating a slider for the player to choose how many lines of the original input data file to skip between lines drawn. As shown in Figure 5. 


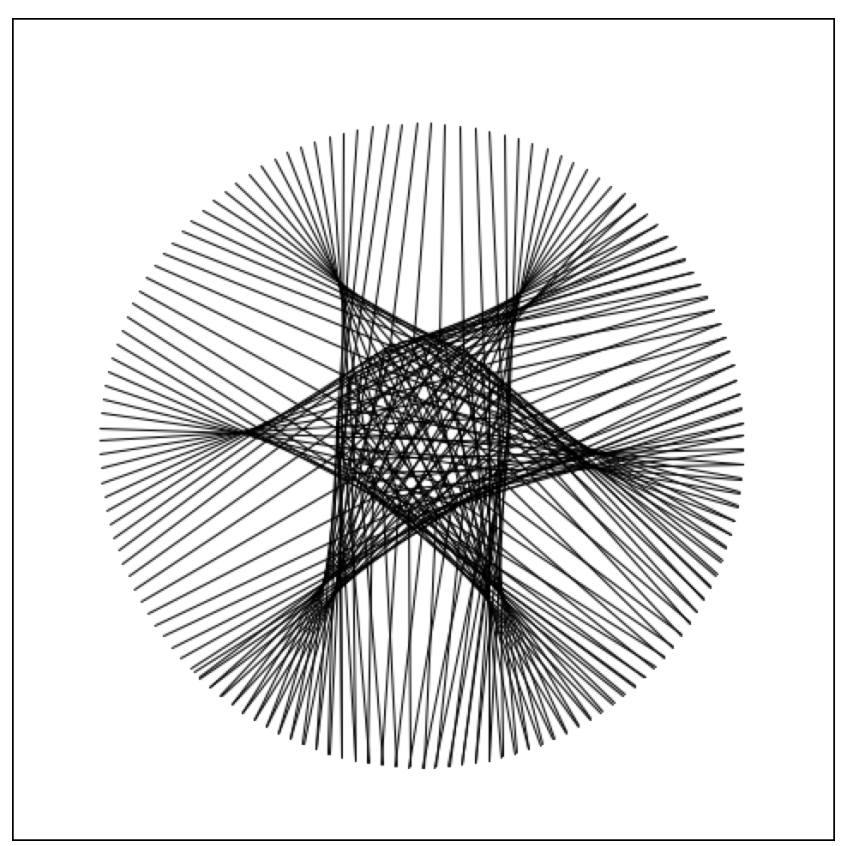

Figure 4: Lines linking Jupiter and Uranus at regular intervals over time

In this simplest of experiments, as the players adjust the time interval between lines drawn and those lines are drawn inside Versum, they begin to see and hear changing symmetrical figures appearing in the data, from the beautiful dense 5-petal structure of the original set, to sparser 3-fold, 4-fold, 5-fold, 6-fold, 9-fold, 17 -fold, etc symmetrical figures. This basic geometric property of varying symmetries may be obvious to mathematicians, but it causes great delight to a first-time explorer and when mapped well sonically, can offer hours of intrigue. Of course, this shifting symmetry pattern happens also in in a similar way with the JupiterUranus link lines when the interval between lines is changed; therefore, this parameter emerges as an interesting and fun potential "knob" for one of the first hardware controller modules.

The player's experience is a dynamic exploration of unfolding information as objects are made visible and audible in Versum's displays (see Figure 6). Perspective (location, camera-pointing) and zoom level within the data is under the control of the player, similar to an in-world avatar game, such as Second Life. In the full module, before starting the navigation or even during navigation, the player will set up their special universe, via an interface such as a mobile device or hardware cockpit control, by choosing from a whole host of variable parameters, such as which planet to include, which moons to include, over what time periods, and at what intervals

Versum enables fluid experimentation with mappings to hundreds of sonic and visual parameters at any moment. Objects can be created, destroyed, and altered in real time either by changing the mappings from the data or by changing navigational perspective. A brief video of this initial proof-of-concept module can be found here: https://drive.google.com/open?id= 1AWaM2C1uLV5m_1m32babRxzy1ttd5kWb Currently, navigation in the Versum prototype is manual and interactive using a 3D space navigator. Our version of Versum uses Supercollider for

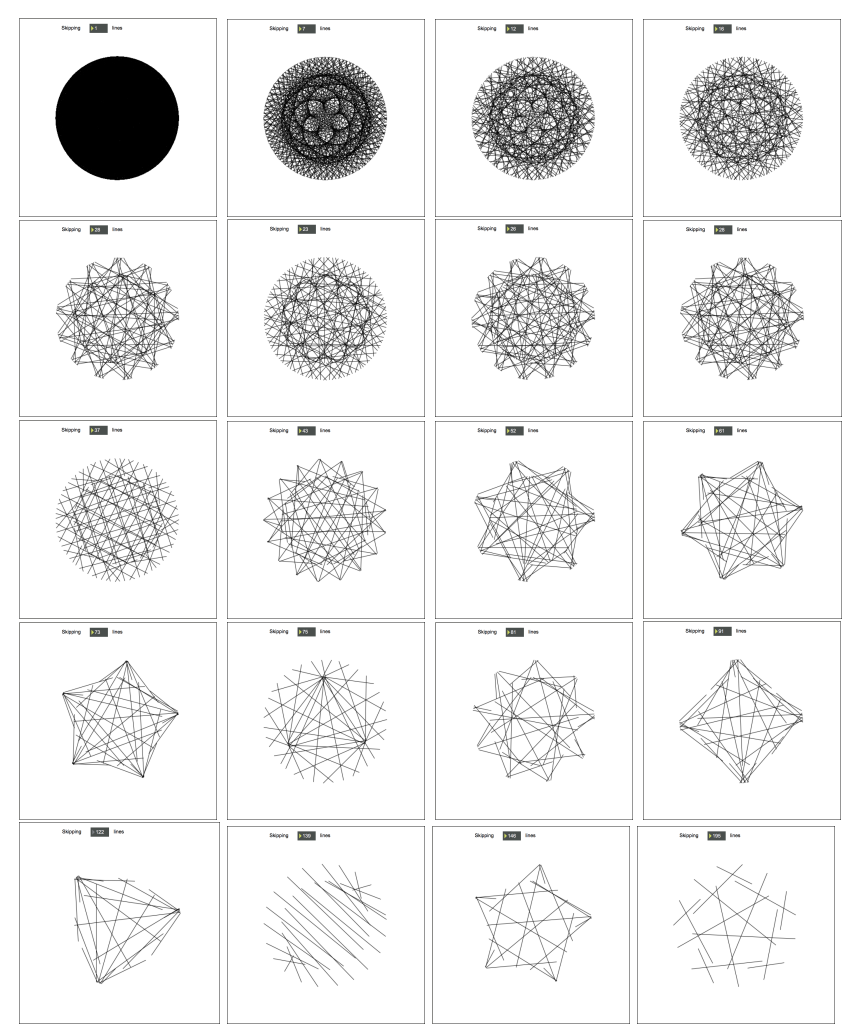

Figure 5: Linklines of Venus and Earth over a period of 100 years with increasing intervals of time between lines, created by varying the number of lines skipped in the database from 0 , representing a line drawn every 4 days (upper left) to every 195th line drawn, representing a line being drawn every 780 days (lower right).

audio synthesis for mappings from the data to audio, such as line length, line angle, regularity of line intersections, distance of intersections from the center of the figure, line start and end points, points where there are multiple intersections, etc. can, themselves, be changed in real time to explore the most compelling mappings for hearing geometric regularity.

\section{CONCLUSION}

Concordia is a musical instrument, inspired by and being built to celebrate the 400th anniversary of Johannes Kepler's 1619 tome Harmonies of the World. The instrument plays scientific data, and is therefore an instrument of investigation and discovery. This new type of instrument - an immersive VR protocol - will enable new types of music and music making. It is built to be massively parallel and collaborative, and to be iterative and cumulative, with plays and discoveries in one moment becoming part of the instrument at the next moment. This extra complexity in the instrument requires extra complexity in its institutional and technical infrastructure, employing new distributed database handling and coordination unimaginable to Kepler, such as blockchain and IPFS.

Still in the initial development stage, a simple proof-ofconcept has been explored using some existing audiovisual tools. For this first module, data from Hartmut Warm's asynchronous, visual data exploration tool has been replicated and in the fully in- 


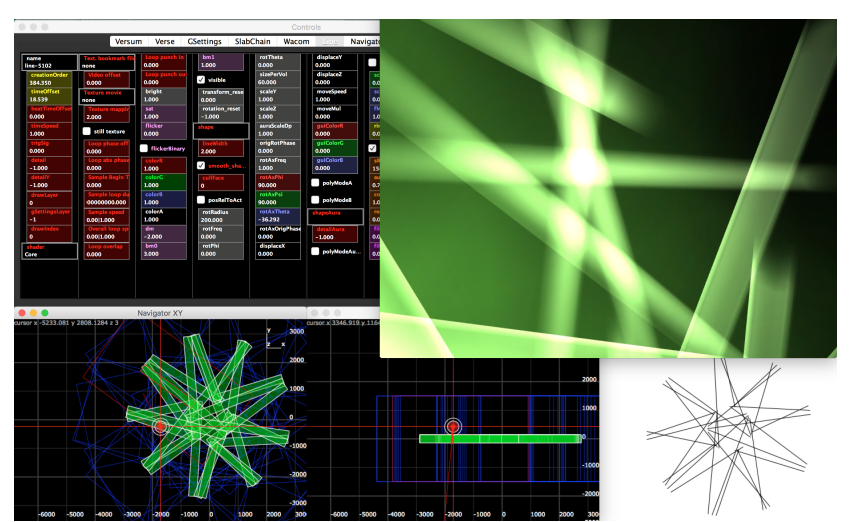

Figure 6: Link lines between Jupiter and Uranus displayed visually and sonically inside Versum.

teractive Versum 3D audiovisual composition environment, such that all mapped sonic and visual properties are data-generated and navigable in real time. The first module is not fully implemented in Virtual Reality, but is an extension into Augmented Reality, allowing an experience of reality at scales not normally accessible to humans. Construction on initial frameworks for the distributed data, computational, and economic architectures has begun, onto which the prototype module will be uploaded for a small group of initial Concordia developers. While the process of creating Concordia will be documented on the blockchain throughout 2018, the four hundredth anniversary of Harmonices Mundi in 2019 will be the official release of Concordia as a functional instrument. Development can continue indefinitely, as contributors add more capabilities to the instrument and upload to the blockchain and distributed platforms, creating an ever expanding, continually updating sonification instrument ecosystem.

\section{FUTURE WORK}

Essentially, nearly all the work on Concordia is still in the future, but development is now occurring at a rapid pace to meet the 2019 anniversary launch deadline. Teams are working in parallel on the various broad areas, including the sonifications, visualizations, immersive media, distributed ledger technologies, education packages for schools and museums, and economics of this new instrument platform.

\section{REFERENCES}

[1] J. Kepler, Epitome of Copernican Astronomy and Harmonies of the World. Prometheus Books, Publishers, 1995, googleBooks-ID: gUJdcFmRUGMC.

[2] H. Warm, Signature of the celestial spheres: discovering order in the Solar System. Forest Row: Sophia, 2010, oCLC: 756267079 .

[3] A. M. Duncan and E. Aiton, Johannes Kepler: Mysterium Cosmographicum (The Secret of the Universe). Abaris Books, 1981.

[4] J. Kepler and C. Baumgardt, "Johannes kepler: life and letters," New York, Philosophical Library [1951], 1951.
[5] V. Galilei, Dialogue on ancient and modern music. Yale University Press, 2003.

[6] T. Barri, "Versum: audiovisual composing in 3d," in Proceedings of The 15th International Conference on Auditory Display (ICAD-2009), Copenhagen, Denmark, 2009.

[7] R. L. Alexander, J. A. Gilbert, E. Landi, M. Simoni, T. H. Zurbuchen, and D. A. Roberts, "Audification as diagnostic tool for exploratory heliospheric data analysis," in The 17th International Conference on Auditory Display (ICAD-2011), Budapest, Hungary, June 2011. [Online]. Available: https://smartech.gatech.edu/handle/1853/51574

[8] M. Ballora, "Sonification Strategies for the Film Rhythms of the Universe," in The 20th International Conference on Auditory Display (ICAD-2014), New York, USA, June 2014. [Online]. Available: https://smartech.gatech.edu/ handle/1853/52075

[9] R. M. Candey, A. M. Schertenleib, D. Merced, and W. L, "Xsonify sonification tool for space physics," in Proceedings of the 12th International Conference on Auditory Display, London, UK, June 2006. [Online]. Available: https://smartech.gatech.edu/handle/1853/50697

[10] N. Leonard, "Sonification: Celestial Data and Poetic Inquiry," in The 16th International Conference on Auditory Display (ICAD-2010), Washington, D.C, USA, June 2010. [Online]. Available: https://smartech.gatech.edu/handle/ $1853 / 50058$

[11] B. J. Tomlinson, R. M. Winters, C. Latina, S. Bhat, M. Rane, and B. N. Walker, "Solar System Sonification: Exploring Earth and its Neighbors Through Sound," in The 23rd International Conference on Auditory Display (ICAD 2017) June 20-23, 2017, Pennsylvania State University, June 2017. [Online]. Available: https://smartech.gatech.edu/ handle/1853/58359

[12] S. Feisst, "18 Negotiating Nature and Music through Technology," in Current Directions in Ecomusicology: Music, Culture, Nature. Routledge, 2015.

[13] W. Ruff, J. Rodgers, and J. Kepler, "The harmony of the world : a realization for the ear of Johannes Kepler's astronomical data from Harmonices Mundi 1619,” 1979.

[14] M. Quinton, I. McGregor, and D. Benyon, "Sonifying the solar system," in The 22nd International Conference on Auditory Display (ICAD-2016). Canberra, Australia, 2016, pp. 28-35. [Online]. Available: http://researchrepository. napier.ac.uk/id/eprint/10317

[15] "Keplerstern.com." [Online]. Available: http: //www.keplerstern.com/signature-of-the-celestial-spheres/ program/

[16] “ssdl.jpl.nasa.gov.” [Online]. Available: https://ssdl.jpl.nasa. gov

[17] C. Scaletti, "Sonification Music," in The Oxford Handbook of Algorithmic Music. Oxford University Press, 2018.

[18] “mi.mu.” [Online]. Available: https://mimugloves.com

[19] C. Bavor, "Virtual and Augmented Realities: Asking the right questions and traveling the path ahead," May 2017. [Online]. Available: http://bit.ly/2pNfVra 
[20] J. Gossmann, "Towards an Auditory Representation of Complexity," in Proceedings of ICAD 05-Eleventh Meeting of the International Conference on Auditory Display, Limerick, Ireland, 2005. [Online]. Available: http: //www.icad.org/Proceedings/2005/Gossman2005.pdf

[21] "ssrn.com." [Online]. Available: http://ssrn.com/abstract= 3174172

[22] S. D. Chris Berg and J. Potts, "Byzantine political economy," Oct. 2017. [Online]. Available: http://bit.ly/2zPjoHg

[23] M. Schedel and E. DeMartelly, "Sustainability of Performing with Technology," in Proceedings of the International Symposium on Electronic Art, 2008.

[24] "https://medium.com/@jason.potts/." [Online]. Available: https://medium.com/@jason.potts/ concordia-a-new-future-economy-of-music-7371f21e917e 